\title{
Spreads and Packings of PG(3,2), Formally!
}

\author{
Nicolas Magaud[0000-0002-9477-4394] \\ ICube UMR 7357 CNRS \\ Université de Strasbourg, France \\ magaud@unistra.fr
}

\begin{abstract}
We study how to formalize in the Coq proof assistant the smallest projective space PG(3,2). We then describe formally the spreads and packings of $\mathrm{PG}(3,2)$, as well as some of their properties. The formalization is rather straightforward, however as the number of objects at stake increases rapidly, we need to exploit some symmetry arguments as well as smart proof techniques to make proof search and verification faster and thus tractable using the Coq proof assistant. This work can be viewed as a first step towards formalizing projective spaces of higher dimension, e.g. PG(4,2), or larger order, e.g. $\mathrm{PG}(3,3)$.
\end{abstract}

\section{Introduction}

Projective incidence geometry [8, 5] is one of the simplest description of geometry, where only points and lines as well as their incidence properties are considered. In addition, in such a setting, we assume that two coplanar lines always meet. There exist some finite and infinite models of projective incidence geometry. Finite projective spaces have been studied extensively from a mathematical point of view (see e.g [12]). Recently [3], we started studying small finite projective planes/spaces from a computer science perspective. We formalized usual projective planes such as $\operatorname{PG}(2,2), \operatorname{PG}(2,3)$ or $\operatorname{PG}(2,5)$ as well as the smallest projective space $\mathrm{PG}(3,2)$ using the Coq proof assistant [7, 1]. We especially focused on proving the synthetic axioms for projective geometry hold in these models. In this paper, we study some of the characteristic subsets of PG(3,2), such as spreads of lines and packings of spreads.

In a three-dimensional setting, the number of points and lines increase rapidly with the order, as shown in Fig. 1. Thus we need to design extremely efficient proof techniques for PG(3,2) if we want our approach to be scalable to projective spaces of higher dimension or larger order. The whole Coq formalization is available online and can be retrieved at: https://github.com/magaud/PG3q. Pointers to specific parts of the development are given throughout the paper.

This paper is organized as follows. In Sect. 2, we show how to formally describe PG(3,2) in Coq using plain inductive types. In Sect. 3, we compute all the spreads and packings of $\mathrm{PG}(3,2)$ and prove some of their properties. In Sect. 4, we present some of the proof optimization techniques we set up in order to achieve the proofs. Finally, in Sect. 5, we outline how this work can be extended to projective spaces of higher dimension or higher order.

\section{Inductive specification of PG(3,2) in Coq}

\subsection{Definitions and operations}

We choose to use two simple inductive types to represent points and lines of PG(3,2). Points are represented by an inductive datatype of 15 constructors without arguments. Lines are represented in the same

Janičić, Kovács (Eds.): Automated Deduction in Geometry (ADG 2021)

EPTCS 352, 2021, pp. 107-115 doi 10.4204/EPTCS.352.12 


\begin{tabular}{|c|c|c|c|}
\hline & \# points & \# lines & \# points per line \\
$P G(2,2)$ & 7 & 7 & 3 \\
\hline$P G(2,3)$ & 13 & 13 & 4 \\
\hline$P G(2,5)$ & 31 & 31 & 6 \\
\hline$P G(2, n)$ & $n^{2}+n+1$ & $n^{2}+n+1$ & $n+1$ \\
\hline \hline$P G(3,2)$ & 15 & 35 & 3 \\
\hline$P G(3,3)$ & 40 & 130 & 4 \\
\hline$P G(3,4)$ & 85 & 357 & 5 \\
\hline$P G(3, q)$ & $\left(q^{2}+1\right)(q+1)$ & $\left(q^{2}+q+1\right)\left(q^{2}+1\right)$ & $q+1$ \\
\hline
\end{tabular}

Figure 1: Numbers of points, lines and points per line depending on the dimension and the order of projective planes and spaces

way using 35 constructors. As there are three points per line, the incidence relation can be represented in a compact way using the match ... with construct of Coq specification language.

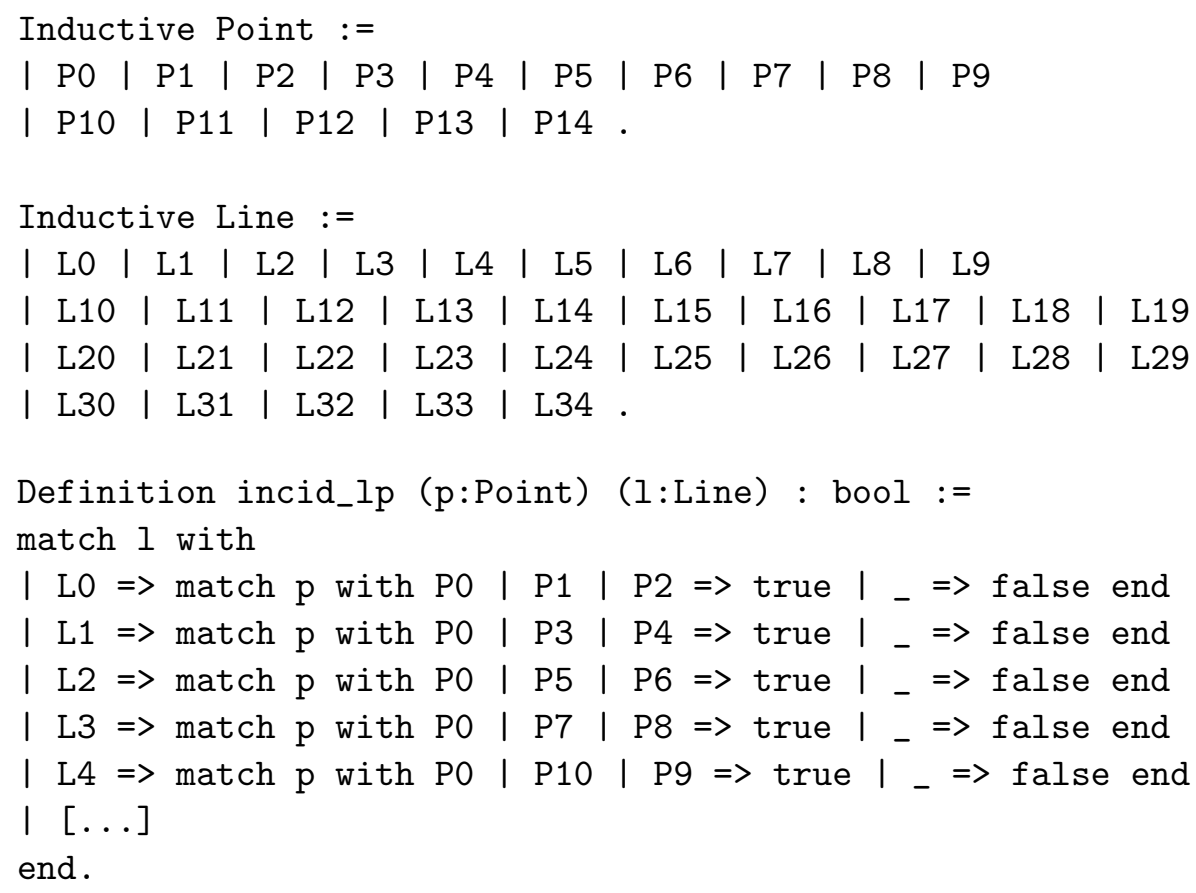

In order to avoid writing too much code in Coq, we choose to build a generator (a simple C program) which takes as input the number of points, the number of lines as well as the incidence relation as a plain file (pg32.txt ${ }^{1}$ which contains for each line of the projective space, the list of the points which are incident to it. Given these three elements, the system automatically builds the inductive data-type for points and lines, the incidence relation. It also defines an artificial order on points and lines based on the index of corresponding points and lines, i.e. $\mathrm{P} 0<\mathrm{P} 1<\mathrm{P} 2<\ldots<\mathrm{P} 14$. The specification generator also builds some auxiliary functions, which will be useful to prove existential statements of the form $\forall l 1 l 2$ : Line, exists $P$ : Point, ....

\footnotetext{
1 https://github.com/magaud/PG3q/blob/master/pg32/pg32.txt
} 


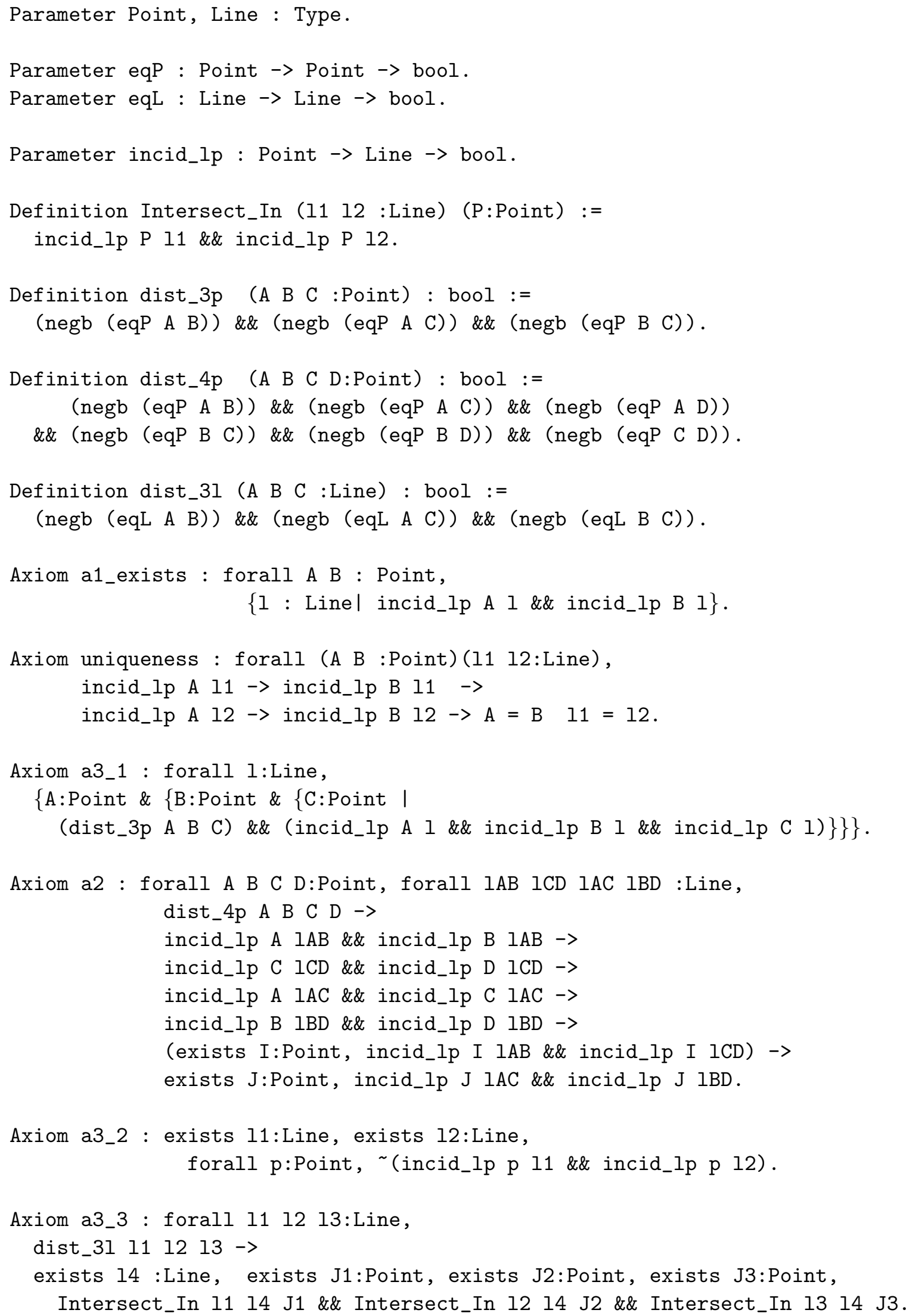

Figure 2: Projective spaces of dimension 3: definitions and properties (pg3x_spec.v) 
Using plain inductive data-types may seem naive. An alternative approach to specify points and lines of $\mathrm{PG}(3,2)$ could be to use finite types ' $I_{n}$ of ssreflect and the mathematical components library [10, 14]. However the main drawback is that ssreflect is designed for formal reasoning rather than computing. Thus checking the incidence between a point and a line is a highly expensive operation, which prevents us from carrying out proofs efficently. Using plain inductive types is much more efficient both to check incidence properties and to perform case analysis. The only drawback is that inductive data-types and functions are huge to write, but this is not that important as we manage to generate these specifications automatically. Overall, our choice is to use the main features of ssreflect, especially the small-scale reflection pattern, but with our own datatypes.

\subsection{Proofs}

Once the projective space $\mathrm{PG}(3,2)$ is described, we check whether all the axioms for projective space geometry hold for this model. This requires proving all axioms of the module defined in https:// github.com/magaud/PG3q/blob/master/generic/pg3x_spec.v and presented in Figure 2. This is pretty straightforward and we try and make it as generic and efficient as possible so that it can be reused for other models of projective space such as $\mathrm{PG}(3,3)$.

\section{Spreads and Packings of PG(3,2)}

\subsection{Definitions}

A spread of $\mathrm{PG}(3, \mathrm{q})$ is a set of $q^{2}+1$ lines which are pairwise disjoint and thus partition the set of points. In $\mathrm{PG}(3,2)$, it corresponds to some sets of 5 lines. A packing of $\mathrm{PG}(3, \mathrm{q})$ is a set of $q^{2}+q+1$ spreads which are pairwise disjoint and thus partition the set of lines. In $\operatorname{PG}(3,2)$, it corresponds to some sets of 7 spreads. As recalled in [2, 6, 13], it is well known that there is only one spread (up to isomorphism) in $\mathrm{PG}(3,2)$ and two packings (up to isomorphism).

\subsection{Generating all Spreads and Packings of PG(3,2)}

Using our external program, we automatically generate all sets of lines of PG $(3,2)$ which are disjoint and cover all the points. As lines contains exactly 3 points, they need to be sets of exactly 5 lines so that all the points of $\mathrm{PG}(3,2)$ are accounted for. We obtain 56 distinct spreads (modulo permutations of the order of the lines involved). We also generate all sets of spreads which are disjoint and cover all the lines. As before, these sets of spreads must have 7 elements, as the number of spreads multiplied by the number of lines in each spread equals the number of lines (35) of PG(3,2). As expected, see Theorem 17.5.6 in [12], we find 240 packings, upto isomorphism. Spreads and packings are grouped in lists. The list of spreads contain 56 spreads, each of them being a list of 5 lines. The list of packings contain 240 packings, each of them being a list of 7 spreads.

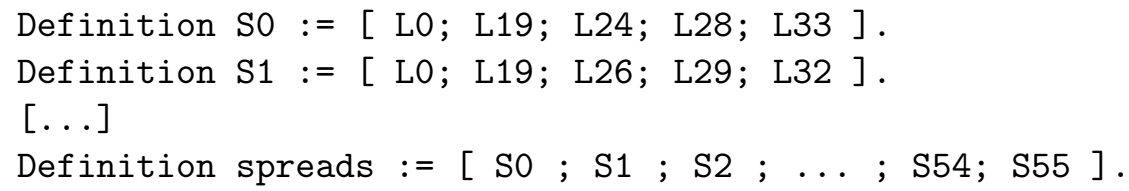




\subsection{Properties}

In Coq, we easily check that the computed spreads and packings verify the properties of spreads and packings.

Spreads can be specified using the following definitions is_partition and is_spread5. The function forall_Point is a finite universal quantification, and forall_Point ( $f$ un $t=>\mathrm{X}$ ) stands for X PO \&\& X P1 \&\& X P2 ... \&\& X P14.

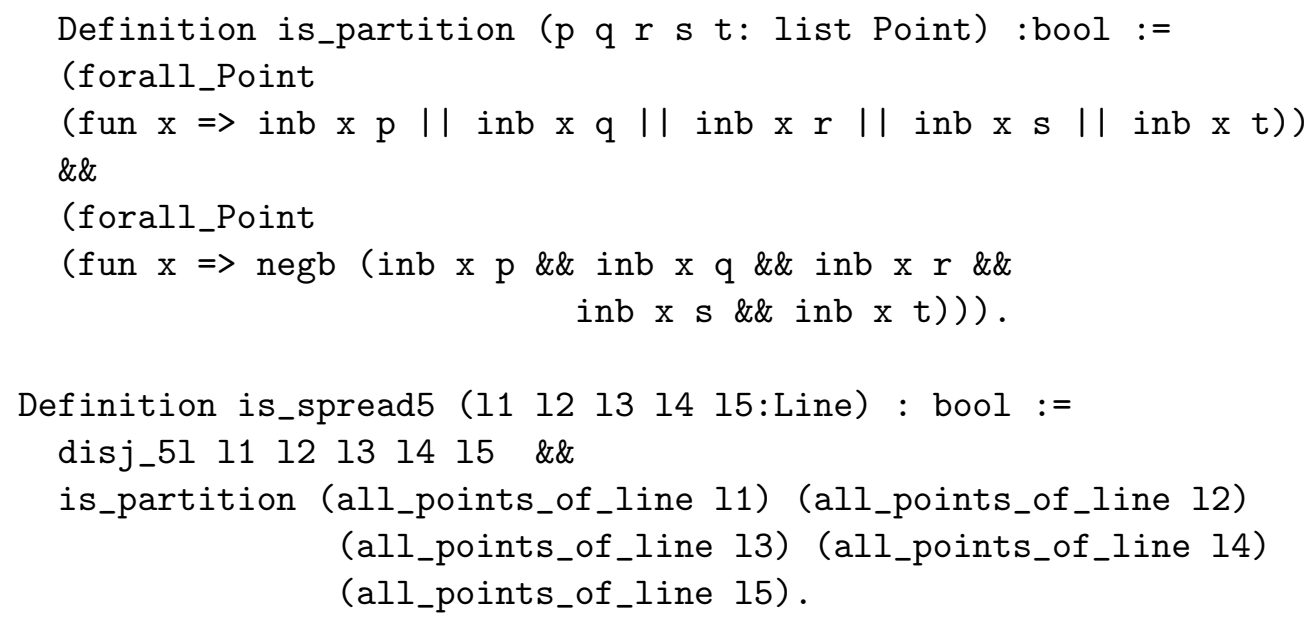

Once these definitions are set, we can prove that the spreads of $\mathrm{PG}(3,2)$ are exactly the ones automatically generated by our external program.

Lemma is_spread_descr : forall 1112131415 , (is_spread5 11121314 15)

In addition, we can prove that all 56 spreads of $\mathrm{PG}(3,2)$ are isomorphic. It can be expressed by stating that there exists a collineation, i.e. an automorphism of PG(3,2) which respects incidence, between any two spreads of PG(3,2).

Lemma all_isomorphic_lemma : forall t1 t2 : list Line, In $t 1$ spreads $\rightarrow$ In $t 2$ spreads $\rightarrow$ are_isomorphic t1 t2.

To prove this statement, we show it is equivalent to simply proving that there exists a collineation (we actually build it) from the $n$-th element of the list to the $(n+1 \bmod 56)$-th element of the lis $1^{2}$.

It is more challenging to check that there are (up to isomorphism) only two packings.

\section{Proof Engineering Techniques}

\subsection{Using bool instead of Prop}

As we work with finite types, equality and the other relations that we use are decidable. We can directly implement such relations as operations producing elements of the boolean datatype bool. This is more convenient than defining them as operations producing elements of type Prop together with a decidability property: $\forall x y,\{x=y\}+\{\neg x=y\}$. This practical approach is inspired by the ssreflect [9] and the mathematical components [14] libraries.

\footnotetext{
${ }^{2}$ https://github.com/magaud/PG3q/blob/master/pg32/pg32_spreads_collineations.v
} 


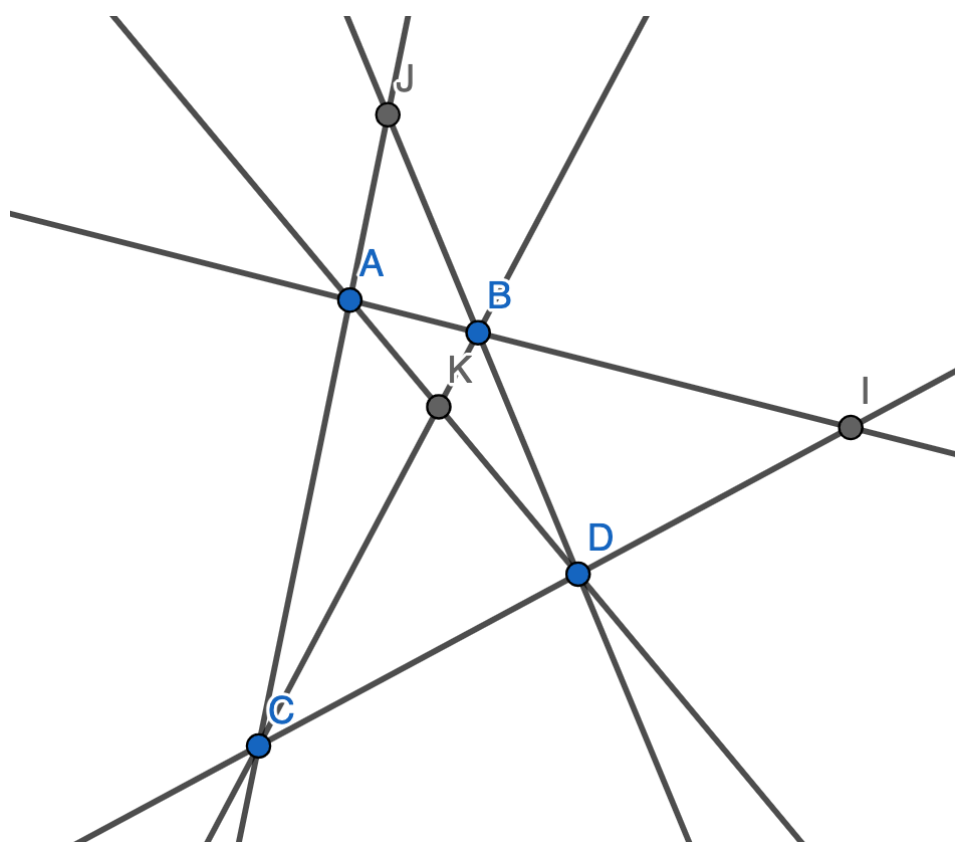

Figure 3: An illustration of the new form of Pasch's axiom used to deal with symmetries

In this setting, logical reasoning (eliminating conjunctions or disjunctions) is a bit more technical. However this makes most proofs much easier to complete by simply computing a boolean value and checking that it is equal to true.

\subsection{Without Loss of Generality}

Most proofs are highly branching. For instance, performing case analysis on all three lines to prove the lemma a3_3 leads to $35^{3}=42875$ cases. In order to make the proof more tractable, we use a tactic named wlog ${ }^{3}$, which implements the without loss of generality principle, as it is described in [11].

This allows to reduce the number of cases to solve explicitly. To use it, we build a virtual order on the points and lines, simply mapping point $\mathrm{Pi}$ (resp. line $\mathrm{Li}$ ) to the value $i$ of its index and extends our statement of the form $\forall l 1, l 2:$ Line, $\ldots$ to $\forall l 1, l 2:$ Line, $l 1<l 2 \rightarrow \ldots$.

Surprisingly, using the without loss of generality tactic forces us to generalize our statement for Pasch's axiom to accommodate all cases, depending on the order in which we consider points $A, B, C$, and $D$, as shown in Fig. 3 .

The usual conclusion of Pasch's axiom

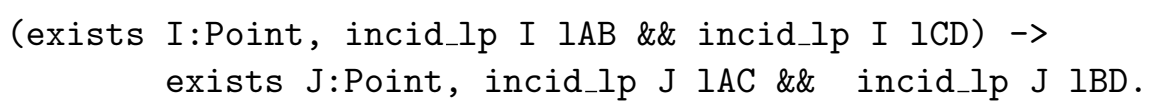

is transformed into a conjunction of two existential properties

...

(exists I:Point, incid_lp I lAB \&\& incid_lp I lCD) $\rightarrow$

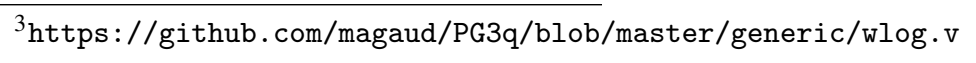


(exists J:Point, (incid_lp J 1 AC \&\& incid_lp J lBD)) $\wedge$

(exists $\mathrm{K}$ :Point, (incid_lp $\mathrm{K}$ lAD \&\& incid_lp $\mathrm{K}$ lBC)).

The tactic wlog was also extremely useful when dealing with spreads and trying to determine inside Coq which sets of lines are actual spreads.

\subsection{Producing Witnesses for Existential Proofs}

In the specification generator, we use a form of skolemisation to write functions which compute the existential variable from the other arguments. For instance, to achieve the proof of lemma a3_3, we automatically build a Coq function $f_{-}$a3 3 3 which, given three lines $l_{1}, l_{2}$ and $l_{3}$ computes a line $l_{4}$ as well as its three intersection points to lines $l_{1}, l_{2}$ and $l_{3}$.

f_a3_3

: Line $\rightarrow$ Line $\rightarrow$ Line $\rightarrow$ Line $*$ (Point $*$ Point $*$ Point)

\subsection{Optimizing proofs}

We design some optimization techniques for generating and checking proof terms. We focus on the current goal, applying some sort of locality principle which means we try to prove a (sub-)goal the very first time we face it. This means sequences of tactics such as

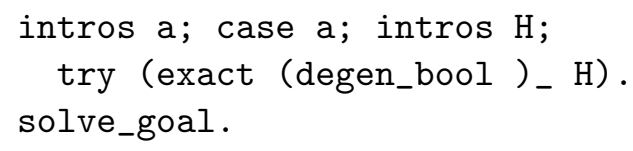

must be replaced by more efficient sequences like

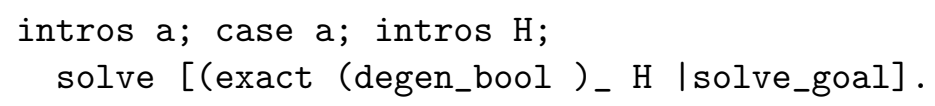

In this simplified example, we try to apply the tactic (exact (degen_bool ) _ H) for a subgoal and then we switch to the next subgoal. Eventually we solve the remaining subgoals using the solve_goal tactic. The idea here is to solve the goal the first time we encounter it. It is achieved by having several possibilities of tactic applications to solve the goal (this corresponds to the solve [t1|t2|t3] syntax. The order of the tactics t1, t2 and t3 can be highly significant as well: we should always call the tactic which is the most successful one on such subgoals first.

As we face a huge number of cases, we need to design extremely efficient prototype tactics on some specific subgoals and apply them automatically to all the subgoals at stake. Fine tuning the tactics rapidly is the key to making the proofs faster to complete.

Finally, Coq provides some sort of task parallelism in the form of the par tactical. It was very useful to deal with all the sub-goals of a proof, once we figure out how to prove the first one. The generic tactic proving the first goal, say mytactic can be easily applied to all sub-goals in parallel (in some cases, we have $35 \times 35=1225$ or more goals to deal with) by simply writing par: mytactic.

\section{Conclusion}

In this work, we manage to formalize in Coq the concepts of spreads and packings. In the context of PG $(3,2)$, we build automatically all the spreads and packings. We can easily verify that these generated 
sets of lines (resp. spreads) are actual spreads (resp. packings). However it remains challenging to verify that they are the only ones. For spreads of PG(3,2), we face a regression issue with Cod ${ }^{4}$ which prevents our proof from being accepted at the Qed step in the current version of Coq.

During this study, we faced case analysis with a huge number of cases as well as debugging proof script with thousands of sub-goals. We propose some proof engineering techniques to make Coq process the files more easily, e.g . by directly providing witnesses for example or by pruning the proof tree by using a without loss of generality principle.

So far, we only address properties and transformations which remain in the same (projective) space. We are currently working on generating specifications of projective spaces automatically in order to easily have a formal description of two different projective spaces and thus to be able to formally describe constructions as the Bruck-Bose construction which allows to build translation planes from projective planes [4].

\section{References}

[1] Yves Bertot \& Pierre Castéran (2004): Interactive Theorem Proving and Program Development, Coq'Art: The Calculus of Inductive Constructions. Texts in Theoretical Computer Science, An EATCS Series, Springer-Verlag, Berlin/Heidelberg, doi:10.1007/978-3-662-07964-5 469 pages.

[2] Anton Betten (2016): The packings of PG(3, 3). Designs, Codes and Cryptography 79(3), pp. 583-595, doi: $10.1007 / \mathrm{s} 10623-015-0074-6$

[3] David Braun, Nicolas Magaud \& Pascal Schreck (2018): Formalizing Some "Small” Finite Models of Projective Geometry in Coq. In Jacques Fleuriot, Dongming Wang \& Jacques Calmet, editors: Proceedings of Artificial Intelligence and Symbolic Computation 2018 (AISC'2018), LNAI 11110, pp. 54-69, doi:10.1007/9783-319-99957-9_4. Available at https://hal.inria.fr/hal-01835493

[4] R.H Bruck \& R.C Bose (1964): The construction of translation planes from projective spaces. Journal of Algebra 1(1), pp. 85-102, doi:10.1016/0021-8693(64)90010-9.

[5] Francis Buekenhout, editor (1995): Handbook of Incidence Geometry. North Holland.

[6] F. N. Cole (1922): Kirkman parades. Bull. Amer. Math. Soc. 28(9), pp. 435-437, doi:10.1090/S0002-99041922-03599-9. Available at https://projecteuclid.org:443/euclid.bams/1183485271

[7] Coq development team (2021): The Coq Proof Assistant Reference Manual, Version 8.13.2. INRIA. Available at http://coq.inria.fr

[8] Harold Scott Macdonald Coxeter (2003): Projective Geometry. Springer Science \& Business Media.

[9] Georges Gonthier \& Assia Mahboubi (2008): A Small Scale Reflection Extension for the Coq system. Technical Report RR-6455, INRIA. Available at http://hal.inria.fr/inria-00258384/

[10] Georges Gonthier, Assia Mahboubi \& Enrico Tassi (2015): A Small Scale Reflection Extension for the Coq system. Research Report RR-6455, Inria Saclay Ile de France. Available at https://hal.inria.fr/ inria-00258384

[11] John Harrison (2009): Without Loss of Generality. In Stefan Berghofer, Tobias Nipkow, Christian Urban \& Makarius Wenzel, editors: Theorem Proving in Higher Order Logics, 22nd International Conference, TPHOLs 2009, Munich, Germany, August 17-20, 2009. Proceedings, Lecture Notes in Computer Science 5674, Springer, pp. 43-59, doi 10.1007/978-3-642-03359-9_3

[12] J. W. P. Hirschfeld (1985): Finite projective spaces of three dimensions. Oxford mathematical monographs, Clarendon Press ; New York : Oxford University Press, Oxford.

\footnotetext{
$\sqrt[4]{\text { https://github.com/coq/coq/issues/13834 }}$
} 
[13] R.H. Jeurissen (1995): Special sets of lines in $P G(3,2)$. Linear Algebra and its Applications 226-228, pp. 617 - 638, doi 10.1016/0024-3795(95)00200-B. Available at http://www.sciencedirect.com/science/ article/pii/002437959500200B. Honoring J.J.Seidel.

[14] Assia Mahboubi \& Enrico Tassi (2016): Mathematical Components. Draft. Available at https:// math-comp.github.io/mcb/. 